\title{
Chapter 4 \\ Sub-Saharan Teacher STEM \\ Professional Development with Digital \\ Ink: Follow-Up (Sub-Saharan Pen-Based \\ Computing Follow-Up)
}

\section{Eric Hamilton, Loide Kapenda, Helena Miranda and Elizabeth Ngololo}

\begin{abstract}
This chapter reports on research activities originally reported at the 2015 WIPTTE conference, the last one held prior to the conference name change. The chapter, entitled "A Model and Research Agenda for Teacher and Student Collaboration Using Tablets in Digital Media Making: Results from Sub-Saharan Workshops" reported on a series of science, technology, engineering, and mathematics (STEM) projects funded by the Kenyan Ministry of Education, Microsoft Research, and the National Science Foundation. The research has continued since then, with additional support from the Namibian Ministry of Education, Arts and Culture, the US State Department's Fulbright Program, and new, multiyear funding from NSF. The chapter describes the evolution of the project and five themes emerging from it, in addition to describing a new four-year effort related to the original paper. The original paper focused on activities in Kenya, Ghana, Uganda, and Namibia. This chapter centers on follow-up activities in Namibia.
\end{abstract}

\subsection{Introduction}

This chapter reports on research activities originally reported at the 2015 WIPTTE conference, the last one held prior to the conference name change. The chapter, entitled "A Model and Research Agenda for Teacher and Student Collaboration Using Tablets in Digital Media Making: Results from Sub-Saharan Workshops"

\footnotetext{
E. Hamilton ( $\bowtie)$

Pepperdine University, Malibu, CA 90263, USA

e-mail: eric.hamilton@ pepperdine.edu

L. Kapenda

Ministry of Education, Arts, and Culture, Windhoek, Namibia

e-mail: loidekapenda@yahoo.com
}

H. Miranda · E. Ngololo

University of Namibia, Windhoek, Namibia

e-mail: hmiranda@unam.na

E. Ngololo

e-mail: engololo@unam.na 
Hamilton et al. (2016) reported on a series of STEM-related projects funded by the Kenyan Ministry of Education, Microsoft Research, and the National Science Foundation. The research has continued since then, with additional support from the Namibian Ministry of Education, Arts and Culture, the US State Department's Fulbright Program in Namibia (Hamilton 2014), and new, multiyear funding from NSF (Hamilton and Culp 2014).

The 2016 CPTTE conference included a paper on pen-based computing interfaces as entrée into learner flow in mathematics (Hamilton 2016). This chapter argued that keyboards are the haptic tools that enable embodied cognition in writing. That is, cognitive and affective processes underlying narrative or prose creation and manipulation are converted into text through the keyboard typing, and that conversion then provisions those processes with the digital tools associated with word-processing or other automation and file management affordances. It was not until the advent of digital inking tools that a similar form of embodied cognition was available for the cognitive and affective processes underlying mathematical or scientific symbol manipulation. While sophisticated software for representing mathematical or scientific notation has greatly simplified publishing in those fields, such software follows and formalizes cognition, rather than facilitating it in real-time.

The ability for school teachers to engage in such embodied cognition in doing mathematics facilitates induction into high engagement, and often flow states, while doing mathematical and scientific operations. Such engagement and flow states are possible without the digital resource of a pen-based computer, of course, but in our research, we have discovered that digitizing such processes provides access to greater collaborative resources and a broader landscape of intellectually challenging activities (Hamilton et al. 2010). In particular, using digital ink interfaces to produce videos that explain or illustrate scientific or mathematical ideas repeatedly has proven profoundly immersive to students and teachers alike. Under a conjecture that growth of mathematical and scientific competencies unfold in an optimal way if individuals are fully absorbed, undistracted, and in full concentration, digital inking in mathematics and science becomes a critical enabler or link behind such growth.

The 2015 paper highlighted challenges facing teachers in low-income nation settings. Teaching is complex and challenging in the US and other wealthy countries. That complexity and difficulty is profoundly intensified in low income nation settings. Some of the most salient limitations might be expected. These include limitations on curriculum materials, especially as low-income countries transition from colonial era curriculum models to independent curricula that reflect context, culture, and customs. Related to this are dynamics associated with language, dynamics that profoundly complicate the challenges low-income countries with multiple dialect and linguistic profiles face. The countries discussed in the earlier paper (Uganda, Namibia, Kenya, and Ghana) each mandate English as the official language of instruction. There is a general consensus in these countries that English proficiency in educational settings are necessary for full entrée into global economics and society. Secondary and often upper primary school instruction typically takes place only in English. Yet recent research confirms that preventing the use of the language of origin in formal learning settings results in lost complex cognition and diminished complex reasoning 
for many students in comparison to peers in multilingual settings that include the language of origin (Yarzebinski et al. 2015).

One of the overarching principles of the work reported here is an understanding that local culture, context, and language is the starting place for education reform initiatives. Digital media-making of the kind that appear in this work is designed to permit code-switching and subtitling in media to scaffold learning and reasoning within a student's language of origin. One way to view this is that pen-based computing opens up new avenues for embodied STEM cognition. The digital inking affordance thus plays a crucial role in connecting formal learning in STEM contexts to flow experiences and to the embedding of digitally-mediated learning in local culture, context, and language.

\subsection{Original Research Method}

When first reported at WIPTTE, the project was taking the form of a hybrid design experiment and phenomenological study, under a three year research grant from the US State Department's Fulbright program (Hamilton 2014). The design iterations were organized around the analysis of three primary data sources. The first involves the artifacts that teachers produced in workshops that engaged teacher participants in pen-based video-making. The second involves structured interviews and reflections with each participant. The third involves debriefing with university and Ministry of Education officials who helped facilitate each workshop. It should be noted that while this three-step design study allows for significant alteration, or macro-redesign, between phases, important "micro-redesign" adjustments took place between each of the workshops that form the basis for this chapter.

\subsubsection{Research Venue: Teacher Workshops with Tablet Computers}

The Ministry of Education and different departments of the University of Namibia organized release time and logistics for five workshops, each held in a region in the northern half of the country along with one in the Omaheke region in east central Namibia (Fig. 4.1). Table 4.1 depicts the attendance at each workshop. The final workshop was held for year three students in secondary mathematics education registered at the Hifikepunye Pohamba Campus of the University of Namibia. The first four workshops were attended primarily by secondary mathematics educators and administrators in their respective regions. The numbers of participants varied in each workshop, from approximately twelve participants to over thirty. The workshops took place over an eight-week period in 2015. Structured interviews and written reflection tools were administered to all participants after the first workshop. 


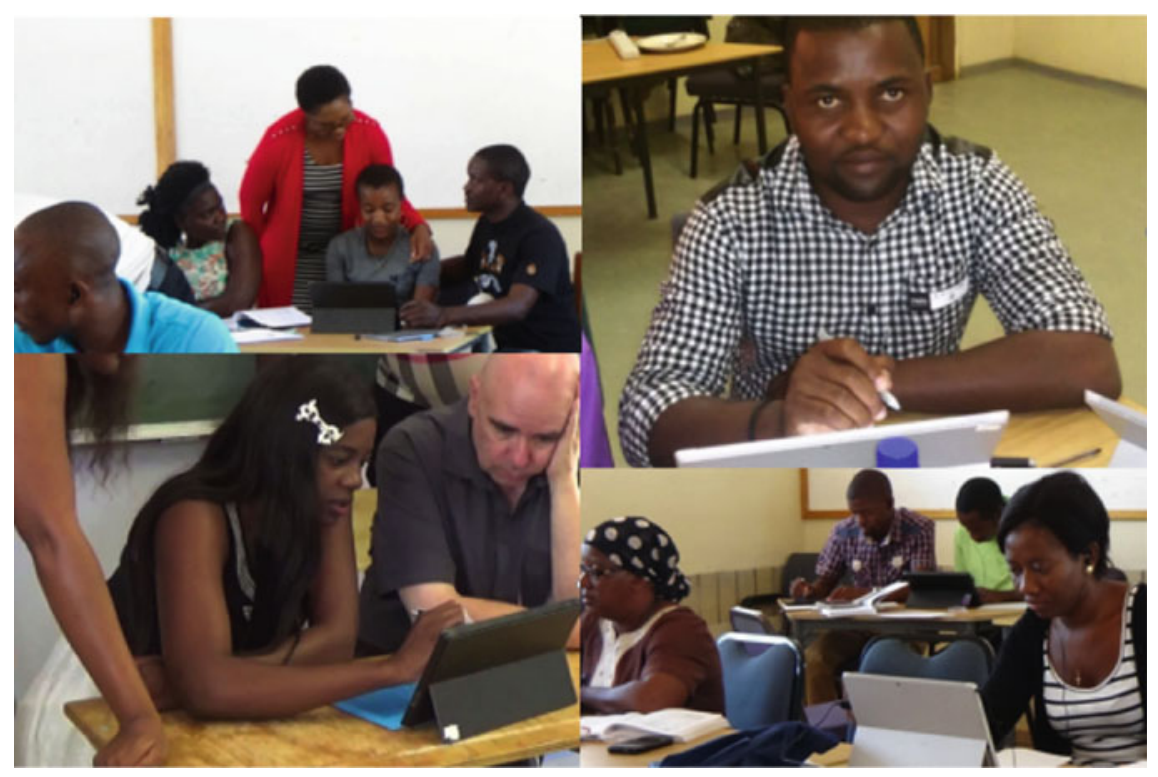

Fig. 4.1 Pen-based media making workshops for teachers in Namibia

Table 4.1 Phase 1 workshop population and data collection

\begin{tabular}{l|l|l|l|l|l}
\hline Workshop & 1 & 2 & 3 & 4 & 5 \\
\hline $\begin{array}{l}\text { Teacher attendees/interviews/reflection } \\
\text { tools (53) }\end{array}$ & $11 / 0 / 0$ & $12 / 12 / 12$ & $9 / 9 / 9$ & $11 / 11 / 11$ & 0 \\
\hline Administrator attendees (7) & 2 & $3 / 0 / 1$ & $1 / 0 / 0$ & $1 / 0 / 0$ & 0 \\
\hline Student (UNAM) attendees (23) & 0 & 0 & 0 & 0 & $23 / 0 / 0$ \\
\hline Learner attendees (5) & $5 / 0 / 0$ & 0 & 0 & 0 & 0 \\
\hline Total & $15 / 0 / 0$ & $15 / 12 / 13$ & $10 / 9 / 9$ & $12 / 11 / 11$ & $23 / 0 / 0$ \\
\hline
\end{tabular}

All participants signed informed consent forms permitting the use of their data and images for research reports. The first workshop included one afternoon by which secondary learners participated with teachers. The workshop duration was site-specific, dependent on local arrangements and attendance and averaging 2.5-3.5 days, with one workshop lasting only for two days.

These workshops were designed to introduce teachers to begin the process of creating and editing instructional videos that incorporate mathematics and science school subject matter. The technology took several forms. For hardware, teachers were provided with pen-based computers (such as Windows-based devices from Fujitsu, Microsoft or Hewlett Packard), Surface Pro devices, or else pen-based writing boards that connect to notebook computers or computers in a classroom lab, (such as Wacom Intuos or Bamboo boards). The writing boards are nearly as functional as 
the tablet computers at about $5 \%$ of the computer price. Working with both provided opportunity to compare the interface usability.

On the software side, teachers were provided with two types of tools. The first was the freely available GeoGebra mathematics visualization software, enabling teachers to prepare powerful, precise, and highly interactive representations of mathematical structures. The second software tool is a suite of programs that allow teachers to record screens into editable and sharable videos. These tools include the Microsoft Journal system, OneNote, and TechSmith's Camtasia. All of the teachers were also provided with sound files, video clips, and a repository of photos that they could use to supplement videos that they designed. With this software, teachers were in a position to produce video-lessons directly on their computers as if they were on a chalkboard or whiteboard, recording and then editing their productions.

Workshops typically began with an introduction to the computer hardware and software, and opportunities for participants to work independently with each. As discussed earlier, one of the most important aspects of working in a pen-based computing environment is an adaptation to the haptic feedback associated with digitizing pen input to computers (Hamilton 2016). For virtually every participant, the workshop represented their first exposure to this interface. The process is not only of adapting to the use of a digitizing pen but connecting the affordance of that interface to their daily instructional practices. In simplified terms, step one is to adapt to the technology, and step two is to use the technology to express one's imagination or content knowledge. Participants were often asked to use the pen to write a short life story that they could share with others.

Next, teachers learned the basic elements of screen-recording. Expressing mathematical or scientific thought on a digital tablet permitted the participants to prepare and record a lesson, using a digital writing tableau such as Windows Journal or OneNote as a digital chalkboard or whiteboard. It is important to understand that this process tacitly obligates countless interactions with the technology and implicitly and explicitly leads teachers to build many new ICT and digital ink competencies.

The process of translating a lesson from a physical whiteboard or chalkboard to a digital ink mode opens an expansive set of affordances to help teachers express their ideas coherently. Teachers report that this is because the process of making a video requires them to treat prepared lessons not as one-off experiences that have no life beyond one class, but rather as reusable artifacts (Hamilton et al. 2010). There is more at stake in expressing the content clearly and concisely. And, every step of the lesson is editable until finalized. In practice, missteps or vague or incorrect explanations in a difficult class lesson usually remain unrepaired-the class ends, and everyone moves on. The editability of a video-mediated by digital ink-means not only that a teacher can give the science or mathematics lesson electronically, but can take the time to make the lesson that as strong and clear as possible. And, as mentioned above, those tools multiply the ways that ideas can be expressed, giving teachers more flexibility in using their imagination to clarify underlying conceptual structures with drawing and scientific and mathematical symbols. Media-making with freehand, digital notation and writing nurtures not only scientific and mathematical coherence but also creativity. 
The workshops thus comprised a mixture of starting from a zero baseline of editing skills to building those skills while implementing them using to express mathematical or scientific concepts using the freehand symbol systems that represent those concepts. This puts upward pressure on each participant to increase their skill base, which in turn gave more flexibility in shaping content for learners. All teachers produced videos of mathematical content, but in the 2-3 day period of instruction, the results were generally quite rudimentary. Additionally, the workshop duration generally did not leave sufficient time for practice in converting the final products into sharable mp4 formats.

\subsubsection{Tablet Computer and Media-Making Workshop Pedagogical Rationale, Continued}

Elsewhere, the rationale for implementing the "media-making" approach has been outlined at some length (Hamilton et al. 2010). An important and distinguishing feature of this effort is that the originating driver or motivation for the workshops is not ICT or pen-based computing, but mathematics (and science) learning. This contrasts with workshops devoted to introducing content-independent ICT tools, such as word processing or spreadsheets. In this case, though, disciplinary content holds priority, over and above the ICT. Yet it turns out that in content-dependent ICT competencies, and pen-based competencies, in particular, that media-making leverages are at least as rich and useful as those that are fostered in more generic ICT exposure.

Our aim in ICT competence is not only to promote technical skills, but for teachers to reflect deeply about content and pedagogy in ways that can advance mathematics and science learning of those they teach. In this regard, teachers consistently report greater attention to mathematical coherence when they plan and create a lesson that will last more than a single class (i.e., have a persistent life as a video); they anticipate students' misconceptions more thoughtfully; they enjoy the deeply creative and imaginative aspects of mixing and remixing media to produce a finished product; and they enter into a deeply absorbed state or flow condition when they are allowed to produce video media (Hamilton 2013, 2017). The digital video editing technology itself, in providing immediate, highly visual, and colorful feedback, affects all of these factors and perhaps most importantly the first-coherence. These findings, outlined below, have also appeared in the US versions of the research. Lack of mathematical coherence has been documented as the most glaring deficiency in mathematics education in the US (Schmidt et al. 2002, 2007). Although there are no known studies focusing directly on the coherence construct in mathematics education in Sub-Saharan Africa, there is no evidence to suggest the situation differs in Namibia. Students acquire skills in manipulating symbols, often with some understanding, but fundamental idea sets remain unconnected, and individual lessons leave students confused with negative feelings directed both at themselves ("I can't do this") and mathematics ("this is not 
useful or understandable") when the cause for misunderstanding may have been a deficient presentation or inadequately prepared teacher-leaving the mathematics symbols unusable for problem solving or operations at what should be the learner's next level of understanding. The process of editing a video benignly coerces coherence, in that the media-maker must think and re-think how each frame connects with the previous frame and with the next frame. This not only induces coherence, but it also deepens the teacher's command of the subject matter. This form of meaningful repetition with a slight variation aligns tightly with the " $R$ " or Repetition principle of Harel's Dualism-Repetition-Need (DNR) framework for deep mathematical reasoning (Harel 2013).

It is in a context of building coherent digital media that this effort sought not simply to integrate pen-based computing and ICT into the classroom, but to do so in a manner that promotes deep thinking and understanding. There are many layers of ICT skills that our learners must acquire for successful participation in an economically growing society. Many of these skills are rudimentary but foundational to more advanced competencies. This particular research focuses on developing the more advanced competencies of helping teachers build digital resources that can help them convey deeper understandings of mathematics and science concepts. These factors led to the original funding proposals to hold these workshops, and the section below furnishes some data that supports this approach.

\subsection{Themes from Research Findings}

This follow-up chapter briefly overviews five critical themes that have emerged from the research. Teachers in this study did indeed perceive pen-based media-making as a potentially powerful tool for more effective teaching. Five important themes recurrently emerged in interviews and written reflections to support their analyses. The concern over a positivity bias is mitigated by the fact that teachers proved able to express these themes differently and with sophistication. They see the pen-based video-making activity as professionally and personally integrative.

\section{Theme 1: More access to technology desired.}

The first theme was mentioned by every teacher and was a factor expected from the outset. It entailed a desire for greater access to hardware and software, and especially tablet computers, such as that employed in the professional development workshops. Many teachers reported an intention to buy costly notebook or tablet computers themselves for continuing to develop and use media-making software tools. All expressed a desire for educational authorities to furnish more technology resources in schools. Resource constraints that face education authorities worldwide are certainly present in Namibia.

Theme 2: Mathematical representation affordances. Teachers routinely were in awe of the digital inking capabilities of their devices and reported in different ways that digital ink permitted them to express mathematical concepts. A recurrent 


\section{theme in teacher interviews involved the usefulness of a media tool that allowed them to represent mathematical ideas quickly and accurately.}

- Media making gives me maximum confidence to teach troublesome concepts like transformations. I will be able to draw all required graphs and learners will be able to hear and see what is happening using media. The best way media making can help me to teach a troublesome topic is by drawing pictures and diagrams while learners are there. I can tell that the workshop left me with enough skills to use media in my class and to share these skills with other teachers at my workplace.

Theme 3: Broader sense of professional well-being. The ability to represent mathematical structure is consistently viewed as a critical factor in making mathematics more accessible. More broadly, the representational affordances of videomaking software are viewed means to promote teachers' creativity, autonomy, and sense of personal empowerment, as shown in the reflections below:

- Digital media has expanded my creativity in a way that I have positive ways of teaching some of the topics that I use to struggle with, to make my explanations self-explanatory.

- This project aroused my eagerness to use tech to improve my students' results. It is even very easy to prepare the lesson the way I want it and leave work behind when I am away. This activity expanded my creativity in a way that now I can create my own videos.

- The (pen-based video-making) expands my creativity a lot and I am able to do much work on my own which will attract learner's interest in the lesson.

- Teaching in itself is an art... I think creating these videos expands creativity.

- Producing materials have altered my sense of personal creativity... (now) I have positive ways of teaching some of the topics that I use to struggle with.

- (I am)... more empowered with the knowledge to produce my own video.

- The instruction to me as a teacher gives me the courage to do my work as an individual and I am ready to support my learners and confident in eradicate the myths of learners who say mathematics is a difficult subject.

- I would have loved this time to be extended just for maybe three months or so...

Theme 4: Teacher-learner collaboration (participatory teaching) potential. In our meetings with the participants, we often observed commentary about the impact of media-rich instructional materials for learners. One topic, though, was of special note here. One relates, in part, to the question of time. What do you think, we asked the teachers, of the idea of learners helping their teachers create videos for peers? Responses from teachers were unanimously positive. Many of them related to the question of youngsters respecting and appreciating one another's perspectives and ways of looking at mathematical ideas. This teacher voiced several important sentiments, expressing intrigue in drawing learners into using media to teach other learners. This teacher's first observation is that learners may, in at least some contexts, comprehend material more readily when provided by others. At the same time, the process of learners helping others is itself an intrinsic learning process - and one that presents content in a way that differs in form from teacher instruction. 
- That (learners helping make videos to benefit other learners) is going to be very interesting. We are saying that if learners are explaining matters to the other learners, then they (will) comprehend easier than teacher teachings, so this is going to be very good. Involving learners in our activities lets them do the work, so, say if the other one is doing this then also the learning process is going to be going up... Others will be listening to them. The way they are talking to each other is not the same way we experience them or how we interact. It is also different.

Another teacher shared similar reflections, stressing the different ways that learners acquire new understandings — and that they can use technology more readily than their teachers:

- I think the learners would be happy (to help teachers make videos). Because they are also involved, they would be able to come up with their own videos also. It would just be easier for them in answering questions on the exam.

\subsubsection{New Project on Participatory Teaching}

It is important to note that Themes 3 and 4 are not overtly related to the digital ink affordances that teachers experienced in their workshops, but those affordances are implicated in the themes. Digital ink gives real-time "voice"- that is, representational functionality to mathematics and teachers (Ellington et al. 2011) that helped forge the sense of professional self-respect and well-being and the potential for participatory teaching.

One of the most important challenges facing teachers in contemporary Namibia is common across most cultures and societies: teachers do not have sufficient time to delve deeply into forming new competencies or adopting novel pedagogies. Time for reflective practice is generally considered a prerequisite to the development of new pedagogies. Yet teachers are hard-pressed to engage in such reflection, let alone devote quality time, and often personal resources to such development. The positive sentiment that teachers across the project expressed in support of collaborating with learners in the media-making process may prove to address the time challenge. Drawing learners into participating in teaching in this way-we call it participatory teaching, a construct substantially larger than peer tutoring or reciprocal teaching - is at the heart of a new project that involves Namibians and counterparts in Kenya, Finland, and the US. Supported by the US National Science Foundation, the project entitled "Research on an International Network for STEM Media Making and Student-led Participatory Teaching" (Hamilton and Culp 2014) builds on the experiences of teachers reported here and will operate over 2017-2020 time frame, exploring how teachers and learners collaborate in media production.

Theme 5: Code-switching as potential strategy. A final theme that emerged in these interviews was the positive sentiments that Namibian participants expressed towards the increasingly strategic area of media-making that involves code-switching 
or multilingual expression. One of the most significant, under-appreciated problems is the policy adopted across many multi-dialect countries, like Namibia, to adopt English as a language of formal education. The policy is pragmatic: educating the next generation in a language that has limited or non-existent use outside of that generation's region or country is a dead-end for full engagement in a broader global society. Recent studies have demonstrated significantly adverse effects of Englishonly school environments for English language learners. English language learners who are not allowed to use their home language in problem-solving produce solutions that fall short of those from peers permitted to move between their original language and English (Finkelstein et al. 2013; Yarzebinski et al. 2015). Essentially, preventing the use of the language of origin informal learning settings results for many students in lost complex cognition and diminished complex reasoning in comparison to peers in multilingual settings that include the language of origin (Webb and Webb 2008). These findings have important implications. They may be even more significant in countries for which development of materials in multiple dialects are commercially infeasible but manageable if carried out locally.

In other words, English-only practices leave important cognition behind. Additionally, in countries that adopt English as an official language but where only a tiny fraction of the teaching population knows English as a first language, the quality of English instruction is suspect. Our interest is in determining teacher attitudes towards producing policy-compliant English language video artifacts with first language captioning and/or oral scaffolds. Every teacher was interviewed except one supported code-switching in their videos.

- You know um... I think for my side, English is the second language for the kid. It is not their first language, and if somebody tells you a story in your own mother tongue you will remember better than if somebody told your story in English. It happens because... if the learner wouldn't understand one word then it is out of their whole system, he will not understand anything.

- Yeah I think (dual language in making videos) would help tremendously. Of course, even if sometimes concepts are very difficult for learners to understand, we as teachers can also take the concepts and explain them in our own languages.

The general sense shared by teacher practitioners reinforces the research findings cited earlier, that important cognition can often only be expressed through a student's original language. The one dissenting participant out of 53 argued that reliance on a student's original language came at the expense of acquiring advanced competencies in English.

This research relates to the implementation of pen-based computing. The use of handwritten input in an intervention that yielded encouragement to incorporate code-switching may seem counterintuitive. Yet enabling code-switching and enabling pen-based input for learning share an epistemic provenance. They both means by which meaning can be represented, shared, and manipulated to solve problems and to produce new knowledge. Permission to speak in one's original language when expressing scientific or mathematical cognition promotes intellectual agency. 
Provision of tools to digitally represent scientific and mathematical symbolism similarly promotes intellectual agency, while also developing technological competences.

\subsubsection{Reflection on Themes of Future Learning Environments}

The future of pen-based computing will depend in part on the degree to which it effectively facilitates progress in emerging visions of the formal and informal educational experience. Since the start of this work reported in the pen-based computing community, the project has adopted the macro-competence framework developed by the Institute for Basic Education at UNESCO (Marope 2017b). The framework is connected to a broader vision of the future of curriculum across the life cycle (Marope 2017a), and identifies seven competences that should be integrated and normative in future learning environments. These include lifelong learning, self-agency, interactively using diverse tools and resources, interacting with others, interacting in and with the world, trans-disciplinarity, and multi-literateness. This chapter does not permit treatment of each of these, but this Sub-Saharan digital ink initiative intersects at least five of them in important ways. In the initiative this chapter reports, pen-based computing proved a catalyst for promoting important dimensions of personal, academic and system growth. It has offered opportunities for much greater intellectual agency. The communication approach enables collaboration, and eventually led a current research effort by which students from multiple countries were able to work together, in an effort supporting computational thinking and personalized learning (Hamilton and Owens 2018).

Acknowledgements This work was carried out under the sponsorship of the Republic of Namibia's Ministry of Education, Art, and Culture, the US National Science Foundation's Cyberlearning, National STEM Digital Library, and Advancing Informal Science Learning Programs, Pepperdine University, and the US State Department's Fulbright Research Program. Their financial sponsorship is gratefully acknowledged.

\section{References}

Ellington AJ, Wilson JH, Nugent JS (2011) Use of tablet pcs to enhance instruction and promote group collaboration in a course to prepare future mathematics specialists. Math Comput Educ 45(2):92

Finkelstein S, Yarzebinski E, Vaughn C, Ogan A, Cassell J (2013) The effects of culturally congruent educational technologies on student achievement. In: International conference on artificial intelligence in education. Springer, pp 493-502

Hamilton ER (2013) Finding creativity and flow in a high-stakes assessment context. Ir Educ Stud 32(1):109-117 
Hamilton E (2014) Theoretical and evidentiary foundation for high performance learning in African schools. Namibia, Fulbright Sub-Saharan research fellowship (\#48140035). http://bit. ly/fulbright-work-proposal-2015-2017. Accessed 10 May 2018

Hamilton E (2016) Digitizing mathematical symbolism through pen and touch technology: a beginning taxonomy of affordances for learner flow in mathematics. In: Conference on pen and touch technology in education, Brown University. 31 March-2 April 2016

Hamilton E (2017) Digitizing mathematical symbolism through pen and touch technology: a beginning taxonomy of affordances for learner flow in mathematics. In: Frontiers in pen and touch. Springer, pp 83-92

Hamilton E, Culp K (2014) Research on an international network for stem media making and student-led participatory teaching. National Science Foundation Award DRL-161284 (Advancing Informal STEM Learning (AISL) Program)

Hamilton E, Harding N, Berque D, Reed R (2010) Tablet computing, creativity and teachers as applied microgenetic analysts: a paradigm shift in math teacher professional development. Impact of pen-based technology on education, pp 47-56

Hamilton E, Kapenda L, Mbasu Z, Miranda H, Ngololo E, Ogwel JC, Sarmonpal S, Stern D (2016) A model and research agenda for teacher and student collaboration using pen-based tablets in digital media making in Sub-Saharan Africa. In: Revolutionizing education with digital ink. Springer, pp 223-230

Hamilton E, Owens AM (2018) Computational thinking and participatory teaching as pathways to personalized learning. In: Digital technologies and instructional design for personalized learning. IGI Global, pp 212-228

Harel G (2013) The Kaputian program and its relation to DNR-based instruction: a common commitment to the development of mathematics with meaning. In: The SimCalc vision and contributions. Springer, pp 437-448

Marope M (2017a) Future competences and the future of curriculum: a global reference for curricula transformation. http://www.ibe.unesco.org/sites/default/files/resources/02_future_ competences_and_the_future_of_curriculum_30oct.v2.pdf. Accessed 10 May 2018

Marope M (2017b) Reconceptualizing and repositioning curriculum in the 21st century: a global paradigm shift. http://www.ibe.unesco.org/en/news/document-reconceptualizing-andrepositioning-curriculum-21st-century. Accessed 10 May 2018

Schmidt W, Houang R, Cogan L (2002) A coherent curriculum. American Education, pp 1-17

Schmidt WH, McKnight CC, Raizen S (2007) A splintered vision: an investigation of US science and mathematics education, vol 3. Springer Science \& Business Media

Webb L, Webb P (2008) Introducing discussion into multilingual mathematics classrooms: an issue of code switching? Pythagoras 2008(1):26-32

Yarzebinski E, Ogan A, Rodrigo MMT, Matsuda N (2015) Understanding students' use of codeswitching in a learning by teaching technology. In: International conference on artificial intelligence in education. Springer, pp 504-513 